\title{
Pro organisierte Suizidbeihilfe
}

\author{
Grischa Merkel • Daniel Häring
}

Online publiziert: 8. März 2015

(c) Springer-Verlag Berlin Heidelberg 2015

Jedes Jahr nehmen sich etwa 10.000 Menschen in Deutschland das Leben. Zur Anzahl assistierter Suizide gibt es keine Statistik, weil die Suizidhilfe in Deutschland zwar strafrechtlich nicht verboten, aber nach den Richtlinien der Ärztekammern auch nicht erlaubt ist. Da Zahlen für Deutschland also fehlen, sei bereits an dieser Stelle ein Blick in die Schweiz erlaubt (wobei die statistischen Daten je nach Quelle variieren): Während im Jahr 1998 rund 40 Fälle von assistierten Suiziden erfasst wurden, waren es im Jahr 2009 schon rund 300. Heute begleiten die Suizidhilfeorganisationen in der Schweiz jährlich rund 830 Personen bei einem assistierten Suizid, viele davon aus Deutschland. Die Thematik ist somit eine bedrückende Realität, vor der die Augen nicht verschlossen werden dürfen und die einer Regelung bedarf.

Von einem verfassungsrechtlichen Standpunkt stellt sich dabei nicht die Frage, ob Sterbehilfeorganisationen zugelassen werden dürfen, sondern ob es gute Argumente gegen ihre Zulassung gibt. Immerhin gehört das Recht zu sterben - und somit nach der hier vertretenen Auffassung auch, dabei Hilfe in Anspruch zu nehmen - zur verfassungsrechtlich geschützten Privatsphäre bzw. persönlichen Freiheit des Suizidenten (so bereits angedeutet im Jahr 1983 von der damaligen Europäischen Menschenrechtskommission im Entscheid $R$. gegen Großbritannien $^{1}$ ). Da die Suizidhilfe strafrechtlich nicht verboten ist, käme eine behördliche Untersagung nur infrage, wenn sich die Organisation mit ihrer Tätigkeit oder ihrem

${ }^{1}$ Europäische Menschenrechtskommission (EKMR) Appl. No. 10083/82 vom 4. Juli 1983, 270 ff.

Der Beitrag „Contra organisierte Suizidbeihilfe“ von Gerrit Hohendorf und Florian Bruns ist unter dem DOI: 10.1007/s00481-015-0341-0 abrufbar.

Prof. Dr. iur. G. Merkel $(\bowtie)$

Juristische Fakultät, Universität Basel,

Peter Merian-Weg 8,

4002 Basel, Schweiz

E-Mail: grischa.merkel@unibas.ch

Dr. iur. D. Häring

Böckli Bühler Partner, Juristische Fakultät, Universität Basel,

Basel, Schweiz 
Zweck gegen die verfassungsmäßige Ordnung richtete. Eine derartige Ausrichtung dürfte in Anbetracht der gesellschaftlichen Diskussion und der Zustimmung von ca. 70\% der Deutschen zum assistierten Suizid aber kaum zu begründen sein. Rekurrieren ließe sich allenfalls auf nicht unumstrittene Gerichtsentscheidungen zu Gewerbeverboten, um die „Würde des Menschen als Gattungswesen" ${ }^{\text {"2 }} \mathrm{zu}$ schützen ${ }^{3}$. Vergleichbar sind sie allerdings schon deshalb nicht, weil Sterbehilfeorganisationen den Vorgang des begleiteten Sterbens der Öffentlichkeit nicht wie in den genannten Entscheidungen im Sinne eines voyeuristischen Spektakels darbieten wollen. Außerdem arbeiten die regelmäßig als Non Profit-Vereine organisierten Sterbehilfeorganisationen meist nicht einnahmeorientiert, sondern decken ihre Auslagen durch Mitgliedsbeiträge, Spenden sowie Gebühren für Freitodbegleitungen. Auch die höchstrichterlichen Entscheidungen zum Schwangerschaftsabbruch ${ }^{4}$ dienen nicht zur Untermauerung eines Verbots. Schon eher ist das Gegenteil der Fall: Gerade am Schwangerschaftsabbruch wird deutlich, dass ein moralisch umstrittener Vorgang sich unter Einhaltung bestimmter Verfahrensvorschriften gesellschaftlich integrieren lässt, ohne Unfrieden hervorzurufen.

Eine klare Regelung in Richtung der Zulassung des assistierten Suizids durch Organisationen bedeutet auch nicht, wie von den Gegnern oft gebetsmühlenartig behauptet, dass damit der Suizid idealisiert würde oder dass vor dem Hintergrund steigender Gesundheitskosten ein sozialer oder wirtschaftlicher Druck auf ältere oder kranke Menschen aufgebaut würde. Die Angst vor dem sog. „Dammbruch“ hat sich in den Staaten, in denen die organisierte Sterbehilfe legal ist, bisher nicht bewahrheitet. Umgekehrt dürfen solche Überlegungen nicht dazu führen, dass Negatives wie Leiden, Krankheit oder Invalidität mittels weltanschaulicher oder religiöser Motive zwingend positiv besetzt werden muss. Als oberstes Gut sollte stets die umfassend informierte Selbstbestimmung eines jeden einzelnen Menschen am Lebensende angestrebt werden.

Wer die Debatte allerdings genau verfolgt, wird bemerkt haben, dass die sog. „Liberalisierung“ der Sterbehilfe im Gegensatz zu der des Schwangerschaftsabbruchs keineswegs darauf abzielt, Menschen umfassend zu informieren und staatliche Hilfe anzubieten, um ihnen in ihrer Situation zu helfen und sie bestenfalls vom Suizid abzubringen. Ganz überwiegend wird von den Abgeordneten des Bundestages ein Verbot der organisierten Sterbehilfe angestrebt. Von einem Bemühen, systematisch all jene Menschen aufzufangen, die sich zurzeit noch hilfesuchend Richtung Schweiz wenden, ist nichts zu lesen. Wer also die Hoffnung hat, mit einer Liberalisierung der Sterbehilfe würden bald Beratungsstellen à la Pro Familia in Deutschland entstehen, vor allem auch, um all jenen, die ihr Leben als nicht mehr lebenswert empfinden, Alternativen zum Sterben aufzuzeigen, sollte diese Hoffnung aufgeben, obwohl gerade dies so wichtig wäre.

Ein Blick in die Schweiz bestätigt dies. Die Sterbehilfe durch Organisationen ist dort zwar nicht ausdrücklich gesetzlich geregelt, wird aber vor dem Hintergrund der anwendbaren Strafnormen als zulässig toleriert. Interessant ist, dass die dort tätigen Organisationen (wie z. B. EXIT oder DIGNITAS) für die hilfesuchenden Menschen sehr viel mehr tun, als Natrium-Pentobarbital zur Verfügung zu stellen; insofern ist bereits die Bezeichnung als

\footnotetext{
${ }^{2}$ Entscheidung des Bundesverfassungsgerichts, amtliche Sammlung (BVerfGE) 87, 209, 228.

${ }^{3}$ Entscheidung des Bundesverwaltungsgerichts, amtliche Sammlung (BVerwGE) 64, 274: Peepshow; Verwaltungsgericht (VG) Neustadt, in: Die Neue Zeitschrift für Verwaltungsrecht (NVwZ) 1993, 98: ,Zwergenweitwurf"; BVerwGE 115, 189: Laserdrome.
}

${ }^{4}$ BVerfGE 39, 1 und BVerfGE 88, 203. 
„Suizidhilfeorganisation“ verkürzt. So leisten die Organisationen z. B. Hilfe beim Verfassen von Patientenverfügungen oder bei der Durchsetzung medizinischer Wünsche bei ärztlichen Behandlungen. Der wichtigste Teil der Tätigkeit liegt aber in der Suizidprävention oder, genauer, in der Suizidversuchsprävention. Neben den in den offiziellen Statistiken erscheinenden vollzogenen Suiziden gibt es eine erschreckend hohe Anzahl von gescheiterten Suizidversuchen (je nach Quelle liegt die Zahl der versuchten Suizide mindestens zehnmal bis zu fünfzigmal höher als jene der tatsächlich „erfolgreich“ ausgeführten). Viele dieser Suizidversuche ziehen sowohl für die Betroffenen als auch deren Angehörige schwerwiegende gesundheitliche und psychische Folgen nach sich. Deshalb engagieren sich die Sterbehilfeorganisationen stark in deren Verhinderung, z. B. mittels Öffentlichkeitsarbeit, Aufklärungskampagnen, dem Aufzeigen von Alternativen zum Suizid, dem Betreiben von Internet-Foren etc.

Besondere Erwähnung verdient beim Blick über die Grenze schließlich, dass im Großteil der Fälle (bis zu 70\%), in denen die Suizidhilfeorganisationen einer Person Hilfe zusichern und ein Arzt nach einer umfassenden Überprüfung der Unterlagen und Konsultation des Patienten seine Zustimmung zur Verschreibung einer letalen Dosis Natrium-Pentobarbital gibt, sich die Menschen gerade nicht suizidieren. Bereits das Wissen, im Notfall auf einen assistierten Suizid zurückgreifen zu können, führt zu einer psychischen Entspannung und zu einer Steigerung der Lebensqualität, welche die Freitodbegleitung oft überflüssig macht.

Nicht nur dies hat das VG Hamburg in seiner Entscheidung vom 6. Februar $2009^{5}$ verkannt, als es eine entgeltliche Suizidhilfe mit Hinweis auf deren „soziale Unwertigkeit“ im Eilverfahren untersagt hat: „Es widerspricht dem Menschenbild des Grundgesetzes, mit dem Suizid und dem Leid von Menschen Geschäfte zu machen." Ob nach dieser Prämisse irgendein Arzt für eine Krankenbehandlung noch Geld nehmen dürfte, sei dahingestellt. Jedenfalls dürfte kein Arzt mit Suizidhilfe Geld verdienen. Dies sieht das VG Berlin in einer Entscheidung vom 30. März $2012^{6}$ offenbar anders. Entgegen der Ansicht der Bundesärztekammer, so das Gericht, müsse dem Arzt ein Ermessensspielraum verbleiben, ob er Suizidhilfe leisten will oder nicht.

Wer Sterbehilfe jedoch nicht den Suizidhilfeorganisationen, sondern lediglich der Gewissensentscheidung einzelner Ärzte überantwortet, muss sich darüber im Klaren sein, dass Ärzte für ihre Beratung, ihre Untersuchungen, An- und Abfahrten sowie Rezeptvergaben ein Honorar nehmen müssen. Die Entgeltlichkeit der Hilfe ist also auch hier ein Thema und könnte sogar ein Anreiz für den Arzt sein, diese zu leisten, anstatt den Patienten an einen evtl. besser qualifizierten Therapeuten zu verweisen. In diesem Zusammenhang muss die Frage erlaubt sein, ob der jeweilige (Haus-)Arzt des Vertrauens überhaupt die Zeit, das Wissen und den Willen hat, angemessen auf die Belange eines sterbewilligen Patienten einzugehen. Immerhin lehnen - im umgekehrten Verhältnis zur Bevölkerung - immer noch gut zwei Drittel der Ärzte in Deutschland die Sterbehilfe ab. Was wäre deren Patienten zu raten, wenn es keine Sterbehilfeorganisationen gäbe?

Hinzu kommt, dass viele Patienten ein Mittel zur Erlösung bei sich zuhause verwahren möchten, um es einzunehmen, wenn und falls sie den Zeitpunkt für gekommen halten. Das birgt vielfältige Gefahren - auch und vor allem dann, wenn die Patienten an ihrer Krankheit versterben, ohne das tödliche Medikament eingenommen zu haben. Bislang sieht kein

$\overline{{ }^{5} \text { Aktenzeichen (Az.): } 8 \text { E 3301/08. }}$.

${ }^{6} \mathrm{Az}$ : VG 9 K 63.09. 
Gesetzentwurf für Suizidhilfe die Überwachung des jeweiligen Medikaments vor, das den Tod herbeiführen soll. Die deutsche Rechtsprechung stellte in der Vergangenheit sogar eine zusätzliche Hürde auf: Wer immer im Augenblick der Medikamenteneinnahme anwesend ist, könnte sich strafbar machen, wenn er nicht augenblicklich Reanimationsmaßnahmen einleitet. ${ }^{7}$ Unklar ist, ob der BGH im Falle organisierter Suizidhilfe an dieser Rechtsprechung festhielte.

Natürlich könnten die Medikamente in den Händen der Ärzte bleiben, bis sie wirklich gebraucht werden, doch besteht dabei die Gefahr, dass sich die Terminkalender von Ärzten mit den individuellen und teilweise dringenden Bedürfnissen todkranker Menschen nicht immer in Übereinstimmung werden bringen lassen. Alternativ stünden prinzipiell zwar Apotheken zur Verfügung, um Medikamente zu verwahren und gegen Rezept herauszugeben. Allerdings ist es einem todkranken Patienten meist nicht möglich, das Medikament selbst abzuholen; und dessen Herausgabe an Dritte, wie etwa Familienangehörige, bleibt mit Unsicherheiten behaftet. Organisationen könnten hier flexiblere Hilfe und eine bessere Kontrolle der Medikamente anbieten.

Nicht unwesentlich erscheint uns schließlich auch, dass nur Organisationen eine adäquate Rechtsvertretung für Betroffene leisten können. Man erinnere sich an den Fall der schwerstkranken deutschen Patientin, die sich beim Bundesamt für Arzneimittel erfolglos um die tödliche Dosis eines Schlafmittels bemühte und ihren Tod schließlich bei einer Sterbehilfeorganisation in der Schweiz fand, und ihren Ehemann, der das Recht auf Suizidhilfe nach ihrem Tod durchzusetzen versuchte. Weil die deutschen Gerichte ihm schon nicht zugestehen wollten, durch die Ablehnung der Behörde beschwert zu sein, müssen sie diesen Fall nun noch einmal prüfen - der Europäische Gerichtshof für Menschenrechte anerkannte die Klagebefugnis des Witwers. ${ }^{8}$ Dem Witwer waren neben einem Schmerzensgeld $26.700 €$ für die Verfahrenskosten zu zahlen. Nur sehr wenige Einzelpersonen, zumal wenn sie schwerkrank sind, verfügen über solche Mittel - ein Verein hingegen könnte seine Mitglieder auch vor Gericht unterstützen.

Unser Fazit: Wer Sterbehilfeorganisationen ablehnt, sollte sich mit der realistischen Alternative eines entsprechenden Verbots auseinandersetzen. Das Bedürfnis an fachlicher Hilfe bei Suiziden wird nicht von Wunderhand verschwinden, gemäß bisherigen Erfahrungen sogar eher noch zunehmen. In den Händen einzelner Ärzte erscheint uns diese Verantwortung schlechter aufgehoben als bei spezialisierten Organisationen. Bis der Staat selbst Beratungsstellen für Betroffene einrichtet und kontrollierte Hilfe für Suizide am Lebensende anbietet, kann deshalb auf Organisationen nicht verzichtet werden.

\footnotetext{
${ }^{7}$ Vgl. dazu Bundesgerichtshof 3. Strafsenat (BGH 3 StR) 96/84 vom 04.07.1984: „Fall Wittig“; aber auch Oberlandesgericht (OLG) München vom 31.07.1987 - 1 Ws 23/87: „Fall Hackethal“; Landgericht (LG) Deggendorf, Beschluss vom 13.9.2013, Az.: 1 KS 4 Js 7438/11.

${ }^{8}$ Entscheid des Europäischen Gerichtshofs für Menschenrechte (EGMR) in Sachen Koch gegen Deutschland vom 19. Juli 2012, Appl. No. 497/09.
} 